\section{Motion Perceptibility and its Application to Active Vision-Based Servo Control}

\author{
Rajeev Sharma and Seth Hutchinson
}

\begin{abstract}
In this paper, we address the ability of a computer vision system to perceive the motion of an object (possibly a robot manipulator) in its field of view. We derive a quantitative measure of motion perceptibility, which relates the magnitude of the rate of change in an object's position to the magnitude of the rate of change in the image of that object. We then show how motion perceptibility can be combined with the traditional notion of manipulability, into a composite perceptibility/manipulability measure. We demonstrate how this composite measure may be applied to a number of different problems involving relative hand/eye positioning and control.
\end{abstract}

Index Terms-Active vision, motion planning, visual servoing.

\section{INTRODUCTION}

In recent years, there has been a growing interest in visual servo control of robotic manipulators [3]. A typical visual servo setup includes a robot and either a fixed supervisory camera (e.g., [7], [14]) or a camera mounted directly on the robot (e.g., [11], [17]). In either case, the image observed by the camera changes due to motion of the robot. Most visual servo controllers compute a control input for the robot that is defined directly in terms of these changes to the image (image-based control), rather than in terms of the absolute position of the robot (position-based control) [3]. Therefore, how the image changes with the motion of the robot has a direct effect on the performance of a visual servo control system. For example, if a large motion of the robot produces very little change in the image, then it may be difficult to use the differential change measured in the image to derive the control input. Hence there is a need for some quantitative measure of the ability to observe changes in the image with respect to the motion of the robot.

In this paper, we introduce, the notion of motion perceptibility, which quantifies the ability of a computer vision system to perceive the motion of objects in its field of view. Although motion perceptibility applies to the motion of arbitrary objects, in this paper, we will focus on applications in which changes in the image are due to motion of the robot. Our definition of motion perceptibility is motivated by earlier research on manipulability [18] and dexterity [6], which are quantitative measures of the relationship between differential change in the end-effector pose relative to differential change in the joint configuration. Manipulability and dexterity, which depend on the particular robot mechanism and its posture, capture a sense of how far the robot is from a singular configuration. For robot control, singular configurations are undesirable since at those configurations the ability to move along one or more dimensions of the task space is lost. Analogous to the motion of a robot end-effector, the "motion" in the image may be near singular configurations with respect to the object that the camera is observing. At such a singular configuration, the vision system will be unable to perceive motions along certain

Manuscript received August 8, 1995; revised May 10, 1996. This paper was recommended for publication by Associate Editor M. Spong and Editor S. Salcudean upon evaluation of the reviewers' comments.

R. Sharma is with the Department of Computer Science and Engineering, The Pennsylvania State University, University Park, PA 16802 USA.

S. Hutchinson is with the Beckman Institute for Advanced Science and Technology, University of Illinois, Urbana, IL 61801 USA.

Publisher Item Identifier S 1042-296X(97)05902-8. dimensions of the task space. The motion perceptibility measure that we introduce in Section III gives a sense of how far the camera/object configuration is from the visual singularities.

Motion perceptibility may be combined in an intuitive manner with the classical manipulability measure to give a composite perceptibility/manipulability measure. We introduce such measures in Section IV, for the cases of nonredundant and redundant systems. Composite perceptibility/manipulability measures can be used as a performance measure for several applications in visual servo control.

Having established a measure of motion perceptibility and composite measures of perceptibility/manipulability, in Section $\mathrm{V}$ we analyze two hand/eye configurations involving an active camera, a four-link redundant planar robot, and a 3-dof PUMA-type robot, and study the variation of the motion perceptibility and the composite perceptibility/manipulability measures. Then, in Section VI we study a series of problems in visual servo control, including optimal camera placement, active camera trajectory planning, and robot trajectory planning. The solution to each problem is illustrated with the help of the hand/eye configurations introduced in Section V. The section ends with a brief discussion of several other potential applications of our motion perceptibility measure. Finally, section VII discusses the advantages and shortcomings of the motion perceptibility measure, followed by some concluding remarks.

\section{Basis of Visual SERvo CONTROL}

As is standard in much of the robotics literature, we use $\boldsymbol{q}$ to represent the configuration of the robot. We will assume that the configuration space is an $n$-dimensional manifold, which implies an $n$ degree-of-freedom robot. The task space of the robot is the set of positions and orientations that the robot tool can attain. For example, if the tool is a single rigid body moving in a three-dimensional workspace, the task space can be represented by $\mathcal{W}=\boldsymbol{R}^{3} \times \mathrm{SO}(3)$. We will use $r \in \mathcal{W}$ to denote an element of the task space, and $m$ to denote the dimension of the task space.

We assign a camera coordinate system with the $x$ - and $y$-axes forming a basis for the image plane, the $z$-axis perpendicular to the image plane (along the optic axis), and with origin located at distance $f$ behind the image plane. Assuming that the projective geometry of the camera is modeled by perspective projection, a point, $\boldsymbol{r}=(x, y, z)$, whose coordinates are expressed with respect to the camera coordinate frame will project onto the image plane with coordinates $(u, v)$, given by

$$
u=f \frac{x}{z}, \quad v=f \frac{y}{z} .
$$

In the computer vision literature, an image feature is any entity that can be extracted from an image, e.g., an edge of a corner. In general, an image feature corresponds to the projection onto the image plane of a structural feature of some object. We define the term image feature parameter to mean any real valued parameter that can be calculated from image features. Image feature parameters may correspond to individual image features or to the relationship between several image features. For example, the image plane coordinates of the projection of a vertex define two image feature parameters. Likewise, the distance in the image plane between the projections of two vertices defines an image feature parameter. Image feature parameters that have been used in the past for visual servo control include the image plane coordinates of points in the image [2], [4], [11], [13], [14], the distance between two points in the image plane and the orientation of the line connecting those two points 


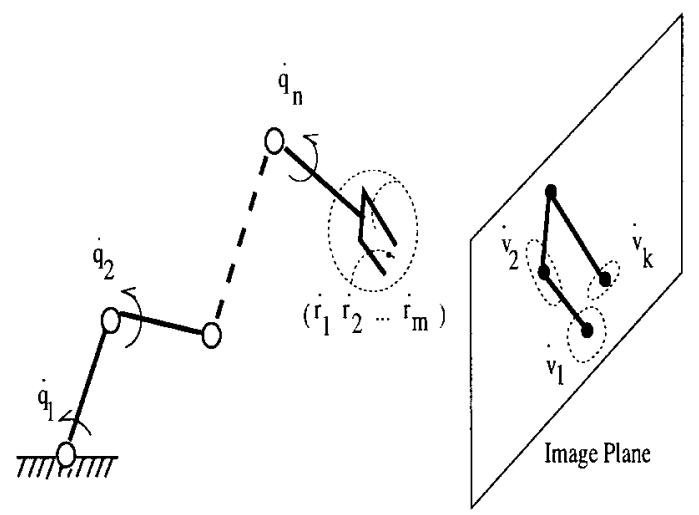

Fig. 1. A schematic representation of the motion perceptibility ellipsoid in the image feature parameter space.

[5], perceived edge length [17], the area of a projected surface and the relative areas of two projected surfaces [17], the centroid and higher order moments of a projected surface [17], [19], the parameters of lines in the image plane [4], and the parameters of an ellipse in the image plane [4]. Discussion of the issues related to feature selection for visual servo control applications can be found in [5], [15], [17]. We denote a vector of $k$ image feature parameters by $\boldsymbol{v}=\left[v_{1} \cdots v_{k}\right]^{T}$.

In visual servo control applications, it is necessary to relate differential changes in the image feature parameters to differential changes in the position of the robot. The image Jacobian captures these relationships

$$
\dot{v}=\boldsymbol{J}_{v} \dot{\boldsymbol{r}}
$$

where

$$
\boldsymbol{J}_{v}(\boldsymbol{r})=\left[\frac{\partial \boldsymbol{v}}{\partial \boldsymbol{r}}\right]=\left[\begin{array}{ccc}
\frac{\partial v_{1}(\boldsymbol{r})}{\partial r_{1}} & \cdots & \frac{\partial v_{1}(\boldsymbol{r})}{\partial r_{m}} \\
\vdots & & \vdots \\
\frac{\partial v_{k}(\boldsymbol{r})}{\partial r_{1}} & \cdots & \frac{\partial v_{k}(\boldsymbol{r})}{\partial r_{m}}
\end{array}\right] .
$$

The image Jacobian was first introduced by Weiss et al. [17], who referred to it as the feature sensitivity matrix. Since its introduction, the image Jacobian has been used in many visual servo control applications, including [4] where it is called the interaction matrix, [11] where it is the $\boldsymbol{B}$ matrix in the state space model of the visual tracking process, and in [2], [5], [13], [19].

Visual servo control applications typically require the computation of $\dot{\boldsymbol{r}}$, given as input $\dot{\boldsymbol{v}}$. There are a number of approaches to deriving $\boldsymbol{v}$ from image data (see, e.g., [1], [11]). Given $\boldsymbol{v}$, when $\boldsymbol{J}_{v}$ is full rank (i.e., $\operatorname{rank}\left(\boldsymbol{J}_{v}\right)=\min (k, m)$ ), it can be used to compute $\dot{\boldsymbol{r}}$. There are three cases that must be considered: $k=m, k<m$, and $k>m$. When $k=m$ and $\boldsymbol{J}_{v}$ is full rank, $\boldsymbol{J}_{v}$ is nonsingular, and $\boldsymbol{J}_{v}^{-1}$ exists. Therefore, in this case, $\dot{\boldsymbol{r}}=\boldsymbol{J}_{v}^{-1} \boldsymbol{v}$. When $k \neq m, \boldsymbol{J}_{v}^{-1}$ does not exist, and pseudoinverse methods may be used (see, e.g., [9]). Here we give only a brief summary.

The solution

$$
\dot{\boldsymbol{r}}=\boldsymbol{J}_{v}^{+} \boldsymbol{\boldsymbol { v }}+\left(\boldsymbol{I}-\boldsymbol{J}_{v}^{+} \boldsymbol{J}_{v}\right) \boldsymbol{b}
$$

where $\boldsymbol{J}_{v}^{+}$is a suitable pseudoinverse for $\boldsymbol{J}_{v}$, and $\boldsymbol{b}$ is an arbitrary vector of the appropriate dimension, minimizes the norm $\left\|\dot{\boldsymbol{v}}-\boldsymbol{J}_{v} \dot{\boldsymbol{r}}\right\|$.

When $k>m$ and $\boldsymbol{J}_{v}$ is full rank, we will typically have an inconsistent system (especially when $\dot{v}$ is obtained from measured image data). In the visual servo application, this implies that we are observing more image features than are required to uniquely determine the object motion $\boldsymbol{r}$. The appropriate pseudoinverse is given by

$$
\boldsymbol{J}_{v}^{+}=\left(\boldsymbol{J}_{v}^{T} \boldsymbol{J}_{v}\right)^{-1} \boldsymbol{J}_{v}^{T} .
$$

When $k<m$, the system is underconstrained. In the visual servo application, this implies that the we are not observing enough image features to uniquely determine the object motion $\dot{r}$. In this case, the appropriate pseudoinverse is given by

$$
\boldsymbol{J}_{v}^{+}=\boldsymbol{J}_{v}^{T}\left(\boldsymbol{J}_{v} \boldsymbol{J}_{v}^{T}\right)^{-1} .
$$

In general, for $k<m,\left(\boldsymbol{I}-\boldsymbol{J}_{v}^{+} \boldsymbol{J}_{v}\right) \neq 0$, and all vectors of the form $\left(\boldsymbol{I}-\boldsymbol{J}_{v}^{+} \boldsymbol{J}_{v}\right) \boldsymbol{b}$ lie in the null space of $\boldsymbol{J}_{v}$, which implies that those components of the object velocity that are unobservable lie in the null space of $\boldsymbol{J}_{v}$.

\section{Motion Perceptibility}

As stated earlier, our goal in this paper is to derive a quantitative value for the perceptibility of an object's (possibly a robot manipulator's) motion, given that the motion is perceived via observation of a set of image features. Here, we introduce such a measure, which we refer to as motion perceptibility. Intuitively, motion perceptibility quantifies the magnitude of changes of the image feature parameters that result from motion of the tool. This is illustrated in Fig. 1. The results presented in this section were inspired by work on manipulability, first presented in [18], and several of the derivations below are analogous to derivations found there.

Consider the set of all robot tool velocities $\dot{r}$ such that

$$
\|\dot{\boldsymbol{r}}\|=\left(\dot{r}_{1}^{2}+\dot{r}_{2}^{2}+\cdots \dot{r}_{m}^{2}\right)^{1 / 2} \leq 1 .
$$

As above, there are three cases to consider. First, consider the case of $k>m$ (i.e., there are redundant image features). We may use (4) to obtain

$$
\begin{aligned}
\|\dot{\boldsymbol{r}}\| & =\dot{\boldsymbol{r}}^{T} \cdot \dot{\boldsymbol{r}} \\
& =\left(\boldsymbol{J}_{v}^{+} \boldsymbol{v}\right)^{T}\left(\boldsymbol{J}_{v}^{+} \boldsymbol{v}\right) \\
& =\dot{\boldsymbol{v}}^{T}\left(\boldsymbol{J}_{v}^{+T} \boldsymbol{J}_{v}^{+}\right) \dot{\boldsymbol{v}} \leq 1 \\
& =\dot{\boldsymbol{v}}^{T}\left(\boldsymbol{J}_{v} \boldsymbol{J}_{v}^{T}\right) \boldsymbol{v} \leq 1
\end{aligned}
$$

Now, consider the singular value decomposition of $\boldsymbol{J}_{v}$, given by

$$
\boldsymbol{J}_{v}=\boldsymbol{U} \Sigma \boldsymbol{V}^{T} .
$$

where

$$
\boldsymbol{U}=\left[\boldsymbol{u}_{1} \boldsymbol{u}_{2} \cdots \boldsymbol{u}_{k}\right], \quad \boldsymbol{V}=\left[\boldsymbol{v}_{1} \boldsymbol{v}_{2} \cdots \boldsymbol{v}_{m}\right]
$$

are orthogonal matrices, and $\Sigma \in \boldsymbol{R}^{k \times m}$. For $k>m$ we have

$$
\Sigma=\left[\begin{array}{ccccc}
\sigma_{1} & & & & \\
& \sigma_{2} & & & \\
& & \cdot & & \\
& & & \cdot & \\
& & 0 & & \sigma_{m}
\end{array}\right]
$$

where the $\sigma_{i}$ are the singular values of $\boldsymbol{J}_{v}$, and $\sigma_{1} \geq \sigma_{2} \cdots \geq \sigma_{m}$.

For this case, the pseudoinverse of the image Jacobian $\boldsymbol{J}_{v}^{+}$is given by (5). Using this with (8) and (9) we obtain

$$
\dot{\boldsymbol{v}}^{T} \boldsymbol{U}\left[\begin{array}{cccc}
\sigma_{1}^{-2} & & & \\
& \sigma_{2}^{-2} & & \\
& & \cdot & \\
& & & \sigma_{m}^{-2}
\end{array}\right] \boldsymbol{U}^{T} \dot{\boldsymbol{v}} \leq 1 .
$$


Consider the orthogonal transformation of $\boldsymbol{v}$ given by

$$
\tilde{\boldsymbol{v}}=\boldsymbol{U}^{T} \dot{\boldsymbol{v}}
$$

Substituting this into (12) we obtain

$$
\sum_{i=1}^{m} \frac{1}{\sigma_{i}^{2}} \tilde{v}_{i} \leq 1 .
$$

Equation (14) defines an ellipsoid in an $m$-dimensional space. We shall refer to this ellipsoid as the motion perceptibility ellipsoid. In Section II, we mentioned that for the case of $k>m$ there are $k-m$ redundant image features. Here, we see that we may use the volume of the $m$-dimensional ellipsoid given in (14) as a quantitative measure of the perceptibility of motion. The volume of the motion perceptibility ellipsoid is given by

$$
K \sqrt{\operatorname{det}\left(\boldsymbol{J}_{v}^{T} \boldsymbol{J}_{v}\right)}
$$

where $K$ is a scaling constant that depends on the dimension of the ellipsoid, $m$. Because the constant $K$ depends only on $m$, it is not relevant for the purpose of evaluating motion perceptibility (since $m$ will be fixed for any particular problem). Therefore, we define the motion perceptibility, which we shall denote by $w_{v}$, as

$$
w_{v}=\sqrt{\operatorname{det}\left(\boldsymbol{J}_{v}^{T} \boldsymbol{J}_{v}\right)}=\sigma_{1} \sigma_{2} \cdots \sigma_{m} .
$$

For the case of $k<m$ we have

$$
\Sigma=\left[\begin{array}{cccc|c}
\sigma_{1} & & & & \\
& \sigma_{2} & & & \\
& & \cdot & & 0 \\
& & & \cdot & \\
& & & \sigma_{k} &
\end{array}\right] .
$$

Since $k<m$, it is not possible to observe motion in all dimensions of the task space. However, the principal axes of the motion perceptibility ellipsoid can be used to determine which motions of the manipulator will be perceptible. This can be particularly useful in hybrid control applications, such as in [2], where only selected degrees of freedom are controlled using visual servo control. In this case, it can be shown that the motion perceptibility ellipsoid has principal axes $\sigma_{1} \boldsymbol{u}_{1}, \sigma_{2} \boldsymbol{u}_{2} \cdots \sigma_{k} \boldsymbol{u}_{k}$, and the motion perceptibility is defined as

$$
w_{v}=\sqrt{\operatorname{det}\left(\boldsymbol{J}_{v} \boldsymbol{J}_{v}^{T}\right)}=\sigma_{1} \sigma_{2} \cdots \sigma_{k} .
$$

For the case of $k=m$ (i.e., when there are no redundant features) we have

$$
w_{v}=\left|\operatorname{det}\left(\boldsymbol{J}_{v}\right)\right| .
$$

To summarize, the motion perceptibility is defined as

$$
\begin{aligned}
& k>m\left(\text { redundant case): } \quad w_{v}=\sqrt{\operatorname{det}\left(\boldsymbol{J}_{v}^{T} \boldsymbol{J}_{v}\right)}\right. \\
& k<m\left(\text { under observed case): } \quad w_{v}=\sqrt{\operatorname{det}\left(\boldsymbol{J}_{v} \boldsymbol{J}_{v}^{T}\right)}\right. \\
& k=m \text { (non-redundant case): } \quad w_{v}=\left|\operatorname{det}\left(\boldsymbol{J}_{v}\right)\right| .
\end{aligned}
$$

The motion perceptibility measure, $w_{v}$, has the following properties, which are direct analogs of properties derived by Yoshikawa for manipulability [18].

- In general, $w_{v}=0$ holds if and only if $\operatorname{rank}\left(\boldsymbol{J}_{v}\right)<\min (k, m)$, (i.e., when $\boldsymbol{J}_{v}$ is not full rank).

- Suppose that there is some error in the measured value of $\boldsymbol{v}$, given by $\Delta \dot{\boldsymbol{v}}$. We can bound the corresponding error in the computed object velocity, $\Delta \dot{\boldsymbol{r}}$, by

$$
\left(\sigma_{1}\right)^{-1} \leq \frac{\|\Delta \dot{\boldsymbol{r}}\|}{\|\Delta \dot{\boldsymbol{v}}\|} \leq\left(\sigma_{m}\right)^{-1} .
$$

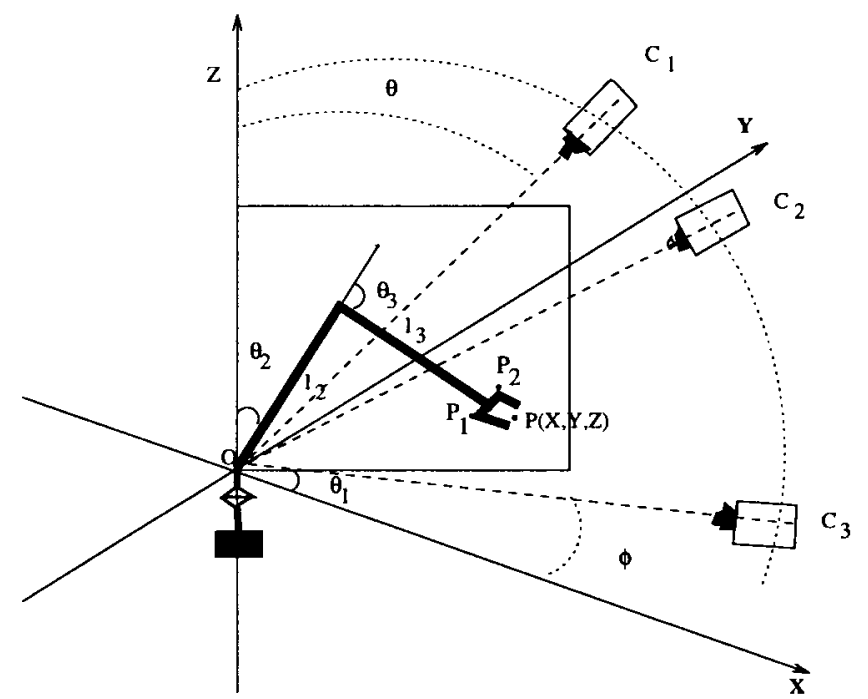

Fig. 2. A hand/eye setup with a 3-dof puma-type robot and a 3-dof active camera.

There are other quantitative methods that could be used to evaluate the perceptibility of motion. For example, in the context of feature selection, Feddema [5] has used the condition number for the image Jacobian, given by $\left\|\boldsymbol{J}_{v}\right\|\left\|\boldsymbol{J}_{v}^{-1}\right\|$. Nelson and Khosla [10] have proposed resolvability. Their concept of resolvability and our concept of motion perceptibility were each inspired by Yoshikawa's work on manipulability [18]. The resolvability ellipsoid is used by Nelson and Khosla [10] mainly as a way to obtain directional properties of the measure. These directional properties are used to derive controllers that actively guide camera-lens motion during task execution. In contrast, motion perceptibility is a scalar quantity, which we use to globally optimize robot performance for an entire task. The only application we consider where the directional property becomes relevant is in Section $\mathrm{D}$, for determining critical directions of hand/eye motion.

\section{A Composite Measure}

The measure of motion perceptibility introduced above can be combined with a measure of manipulability to give a composite measure of how good a camera/robot setup is with respect to visionbased control. The system designer is often free to choose where in the robot workspace a particular task is to be performed. In such cases, it is desirable to choose the robot trajectory and the camera location so that both motion perceptibility and manipulability are optimized.

Manipulability as introduced in [18], is defined by

$$
w_{r}=\sqrt{\operatorname{det}\left(\boldsymbol{J}_{r} \boldsymbol{J}_{r}^{T}\right)}
$$

where $\boldsymbol{J}_{r} \in \boldsymbol{R}^{m \times n}$ is the manipulator Jacobian matrix, and

$$
\dot{\boldsymbol{r}}=\boldsymbol{J}_{r} \dot{\boldsymbol{q}}
$$

is the expression relating the robot joint velocities $\dot{q}$ to the velocity of the end effector $\dot{\boldsymbol{r}}$.

The simplest approach to combining the measure of manipulability and motion perceptibility is to compose the forward kinematic map of the robot with the image mapping $v$. Combining (22) and (2) we obtain

$$
\dot{v}=\boldsymbol{J}_{v} \boldsymbol{J}_{r} \dot{\boldsymbol{q}}
$$

Let $\boldsymbol{J}_{v} \boldsymbol{J}_{r}=\boldsymbol{J}_{c} \in \boldsymbol{R}^{k \times n}$ denote the composite Jacobian matrix. The composite Jacobian $\boldsymbol{J}_{c}$ relates differential changes in configuration of the robot to differential changes in the observed image feature 
parameters. In particular, consider the set of all joint velocities $\dot{q}$ such that

$$
\|\dot{\boldsymbol{q}}\|=\left(\dot{q}_{1}^{2}+\dot{q}_{2}^{2}+\cdots \dot{q}_{n}^{2}\right)^{1 / 2} \leq 1 .
$$

For the case when $n \leq m$, that is a nonredundant robot, for $k>m$ it can be shown (using derivations similar to those given in Section III) that the corresponding set of image feature parameter velocities is given by the set of all $\boldsymbol{v}$ such that

$$
\dot{\boldsymbol{v}}^{T} \boldsymbol{J}_{c} \boldsymbol{J}_{c}^{T} \dot{\boldsymbol{v}} \leq 1
$$

It can be shown (again, using derivations similar to those given in Section III) that, for the nonredundant robot, (25) defines an ellipse whose volume is proportional to $w$, where

$$
w=\sqrt{\operatorname{det}\left(\boldsymbol{J}_{c} T \boldsymbol{J}_{c}\right)} .
$$

Thus, we once again obtain a measure of motion perceptibility. However, in this case, we measure the perceptibility of the joint velocity rather than the perceptibility of motion of the robot tool. In this case, if a particular joint motion cannot be perceived, there are two possible causes: the vision system cannot observe the corresponding motion of the tool (a singularity in the image Jacobian), or the robot is in a configuration in which certain tool velocities are not possible (a singularity in the manipulator Jacobian). For redundant robots, clearly $w=0$, since $\operatorname{rank}\left(\boldsymbol{J}_{c}^{T} \boldsymbol{J}_{c}\right) \leq m<n$ and $\boldsymbol{J}_{c}^{T} \boldsymbol{J}_{c} \in \boldsymbol{R}^{n \times n}$.

The above formulation of the composite measure is not applicable to a redundant robot. Therefore, we formulate an alternative composite measure that is also applicable to the redundant case, as

$$
w=w_{v} w_{r} .
$$

Thus the motion perceptibility $w_{v}$ and the manipulability $w_{r}$ are first computed separately and then multiplied to give the composite measure. Since $w_{v}$ and $w_{r}$ can be computed for the general case, as discussed earlier, (27) forms a general way of combining the motion perceptibility and the manipulability into a single scalar measure.

Another alternative for combining motion perceptibility with manipulability is to define a composite measure that is the weighted sum of the two measures

$$
w=k_{v} w_{v}+k_{r} w_{r}
$$

where $k_{v}$ and $k_{r}$ are constants that allow us to weight the relative importance of motion perceptibility and manipulability. The advantage to this formulation is that it allows us to independently consider motion perceptibility and manipulability, and thus is applicable to the case of redundant manipulators. This decoupling is also useful for considering simultaneously the tasks of robot trajectory planning and camera trajectory planning, with additional constraints for steering both $w_{v}$ and $w_{r}$ away from zero. However, in this case, $w_{v}$ and $w_{r}$ need to be normalized and scaled [6] to make the composite measure useful. In the examples that we consider next, we use the product rule for obtaining the composite measure.

\section{Analysis of Visual Servo Setups}

In this section, we investigate the behavior of motion perceptibility, $w_{v}$, and of composite perceptibility/manipulability, $w$, for two hand/eye setups involving an active camera with a redundant and a nonredundant robot. In particular, we show how $w$ and $w_{v}$ vary with respect to a number of parameters, including configuration parameters of the camera, and configuration parameters of the robot. This serves to provide insight into the nature of these measures, and leads to the applications presented in Section VI.

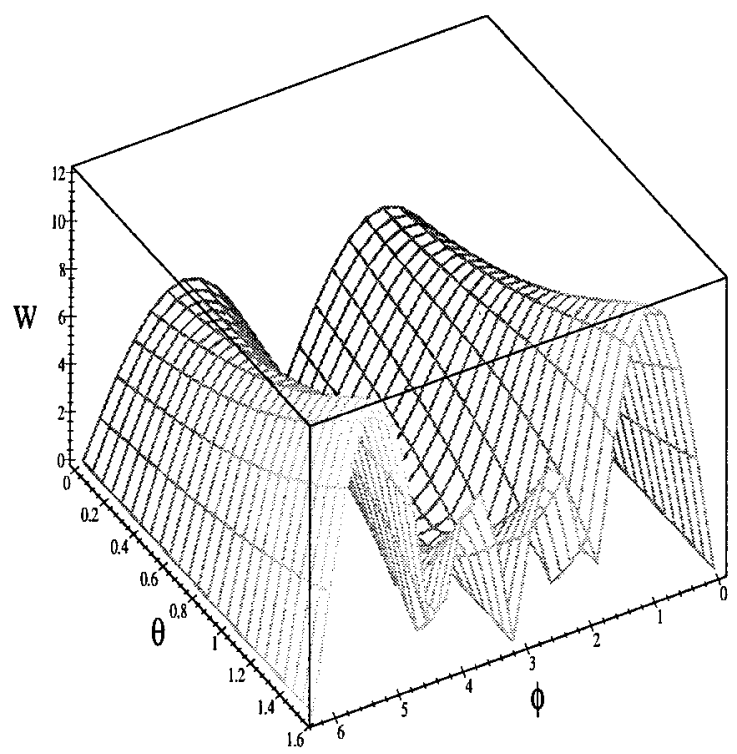

(a)

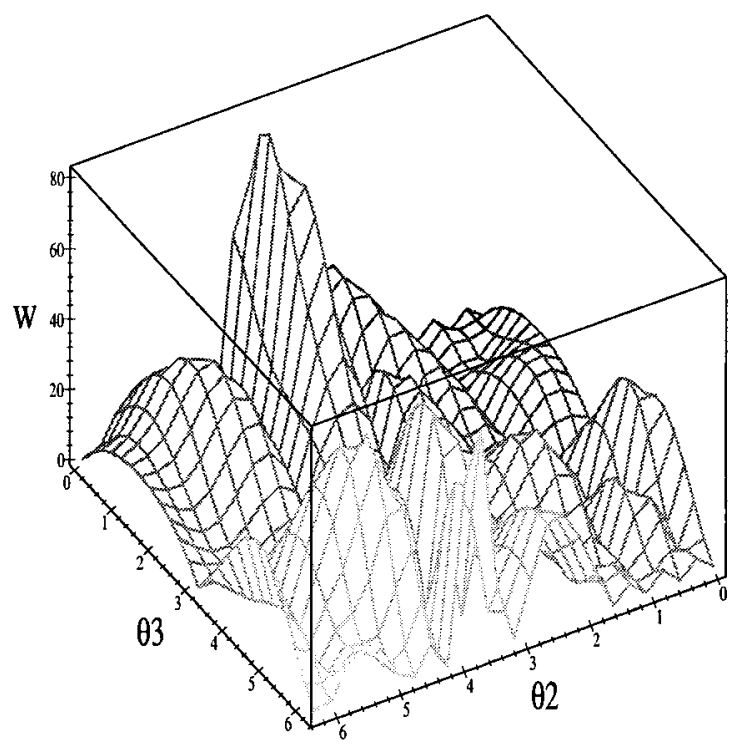

(b)

Fig. 3. Variation of the composite perceptibility/manipulability $w$ with (a) different camera positions $(\phi, \theta)$ for a given robot configuration, ( $\theta_{1}=\pi ; \theta_{2}=\pi / 3 ; \theta_{3}=\pi / 4$ ), and (b) different robot configurations for a given camera configuration $(\phi=\pi / 3, \theta=\pi / 3)$.

\section{A. A 3-dof PUMA-Type Robot}

In this section we consider a 3-dof Puma-type robot, with the main three joints, and a movable camera (Fig. 2). We define the task space to be the set of positions that the robot tool can reach, parameterized by $(x, y, z)$. Thus the robot is used in a nonredundant manner, and in this case the composite perceptibility/manipulability measure is obtained directly from the image and manipulator Jacobian (26). The configuration space of the robot can be parameterized by the three joint angles, $\left(\theta_{1}, \theta_{2}, \theta_{3}\right)$. We constrain the active camera to have only three degrees of freedom, always pointing toward the origin of the world coordinate frame, as illustrated in Fig. 2. The camera configuration can then be parameterized by the spherical coordinates $(R, \theta, \phi)$ of its image center.

To achieve the necessary servo control, we track three image features. Here we use the feature vector $\left[u_{1}, v_{1}, u_{2}\right]^{T}$, where $u_{i}, v_{i}$ 


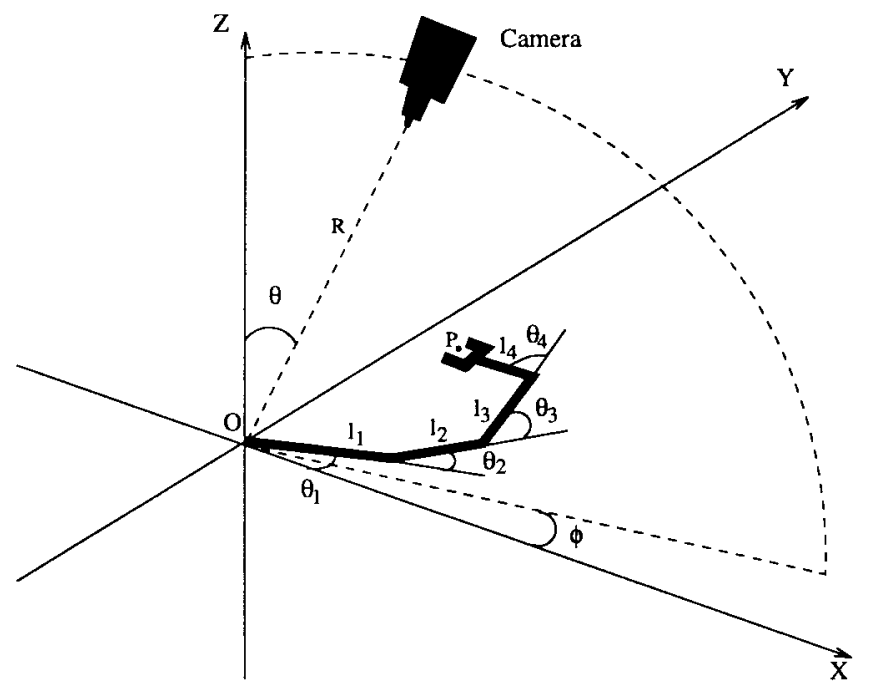

Fig. 4. A hand/eye setup with a 4-dof redundant planar robot and a 3-dof active camera.

are the image plane coordinates of a point $P_{i}, i=1,2$, on the robot end effector (note that we do not use $v_{2}$ in this example). The points $P_{1}$ and $P_{2}$ are illustrated in Fig. 2. The calculation of $\boldsymbol{J}_{v}$ and $\boldsymbol{J}_{r}$ is straightforward, and are omitted here. The Jacobian $\boldsymbol{J}_{c}$ is computed as the product $\boldsymbol{J}_{v} \cdot \boldsymbol{J}_{r}$ and the composite perceptibility/manipulability measure is computed using (26). Fig. 3(a) illustrates the variation of the composite measure, $w$, with a variation of the camera position, for a fixed configuration of the robot while Fig. 3(b) illustrates the variation of $w$ for different robot configurations for a fixed camera configuration. This figure clearly illustrates the strong dependence of $w$ on the relative position of the camera and manipulator. The camera parameter $\theta, \phi$, as well as the robot configuration parameters $\theta_{1}, \theta_{2}, \theta_{3}$ are given in radians. For both the examples, the distance of the camera from the origin, $R$ is set to 50 units while the focal length of the camera is 20 units. In both the figures, only two of the parameters are shown to vary while the others are fixed; this helps in ease of visualizing the variation.

Clearly, in a robot task with an active camera, the "trough" on the surface plots (when the values of $w$ are nearly zero) in Fig. 3 should be avoided since the corresponding configuration may be near kinematic or visual singularities. Section VI will consider the formalisms that help avoid these singular measures by optimizing $w$ in different visual servoing tasks.

\section{B. A Four-Link Planar Redundant Robot}

In this section, we consider a hand/eye setup involving a four-link planar arm, and a movable camera. The task space is parameterized by $(x, y)$, the position in the plane of the robot's tool center, $P$, thus the robot is used in a redundant manner. The configuration space of the robot can be parameterized by the four joint angles, $\left(\theta_{1}, \theta_{2}, \theta_{3}, \theta_{4}\right)$. As in the previous example, the active camera has three degrees of freedom, pointing toward the origin of the world coordinate frame, as illustrated in Fig. 4. The camera configuration is parameterized by the spherical coordinates $(R, \theta, \phi)$ of its image center.

For position visual servo control of the planar robot, two image feature parameters are sufficient, as described in Section II. Here, we use the image coordinates $(u, v)$ of the point $P$. We assume that the end effector is always in the field of view of the camera, so that the image of $P$ can be extracted. Issues related to the use of an active camera to help keep a point in the field of view have been discussed, for example, in [12] and [16].

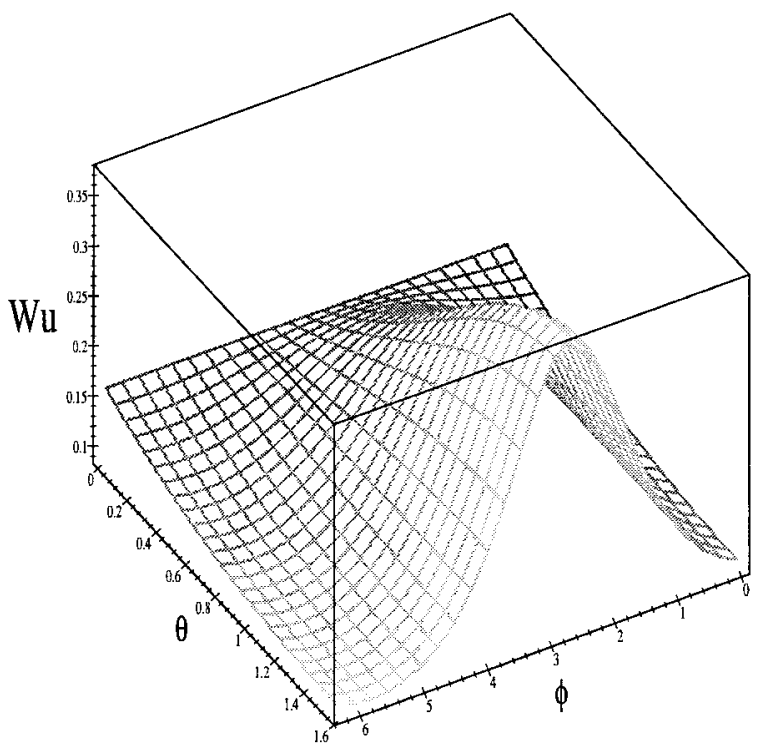

(a)

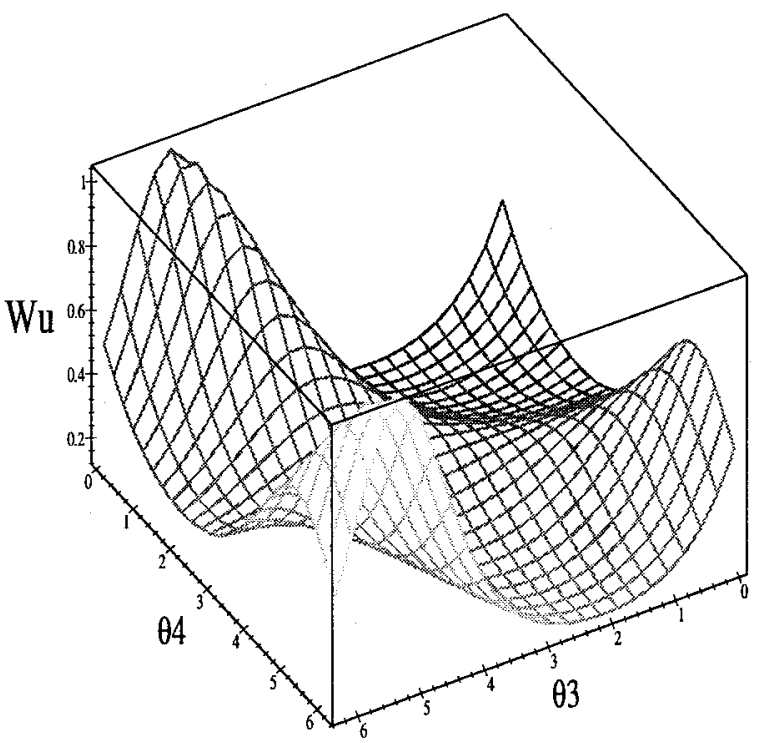

(b)

Fig. 5. Variation of the motion perceptibility $w_{v}$ with changes in (a) camera configuration and (b) robot configuration.

For the case of the redundant robot, we first separately derive $w_{v}$ and $w_{r}$, as discussed earlier. Again, computing $\boldsymbol{J}_{r}$ and $\boldsymbol{J}_{v}$ is fairly straightforward [9] and is omitted here. $w_{v}$ is computed as $\operatorname{det}\left(\boldsymbol{J}_{v}\right)$, since $k=m$, but for computing $w_{r}$, the form $\sqrt{\operatorname{det}\left(\boldsymbol{J}_{r} \boldsymbol{J}_{r}^{T}\right)}$ is used since $n>m$. An important thing to note is that $w_{v}$ depends on both the camera parameters as well as on the robot parameters. This dependence is reflected in the plots in Fig. 5, which shows the variation of $w_{v}$ with the camera parameters $(\theta, \phi)$ in (a) and the variation of $w_{v}$ with the robot configuration parameters $\left(\theta_{3}, \theta_{4}\right)$ in (b). The variation of the robot manipulability, $w_{r}$, with two of the robot joint parameters is shown in Fig. 6 .

The composition of the motion perceptibility and the manipulability is done using the product rule of (27). The variation of the composite measure with two of the camera parameters is shown in Fig. 7(a), while its variation with two of the robot joint parameters is shown in Fig. 7(b). Depending on a particular application, one or more of these parameters can be used for "steering" the hand/eye setup away from configurations near the singularities (that correspond to low values of 


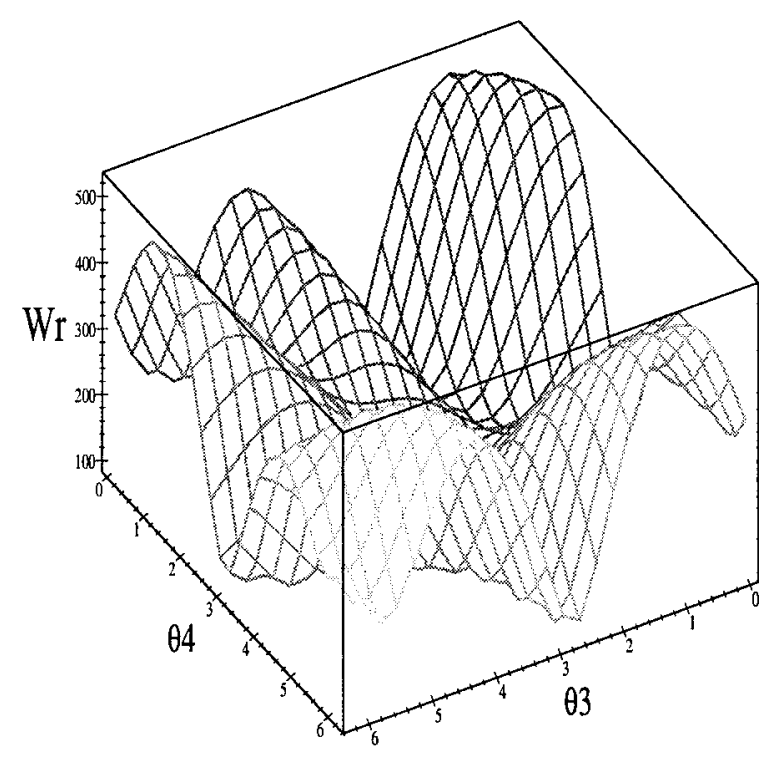

Fig. 6. Variation of the manipulability $w_{r}$ with the changes in the robot configuration.

$w)$. For example, in some task, the null space of the redundant robot (see discussion in Section II) can be used to optimize the value of $w$. Some of these applications are considered next.

\section{ApPLICATIONS}

In this section we show how the composite perceptibility/manipulability measure and the motion perceptibility can be applied for improving different aspects of the visual servo control with a possibly active camera. The optimization problems thus posed, are illustrated with the help of the servo control setups introduced in the previous section.

\section{A. Optimal Camera Positioning}

Here we will address the problem of optimal camera placement for a fixed camera system that is used to control a robot performing some predetermined motion, e.g., a robot grasping an object. We briefly formalize the problem of optimal camera placement with respect to our composite measure of perceptibility/manipulability, $w$. With respect to perceptibility, we do not address issues related to other camera placement criteria, e.g., occlusion, field of view, depth of field, focus, etc. These issues have been addressed in [12], [16]. In fact, the methods presented in [16] produce a set of constraints on camera position and orientation that could be used as additional constraints in the optimization process discussed here.

In order to perform the optimization, we posit the following performance functional

$$
\psi\left(\boldsymbol{q}_{c}\right)=\int w\left(\boldsymbol{r}, \boldsymbol{q}_{c}\right) d t
$$

where $\boldsymbol{q}_{c}$ is a vector of camera parameters that determines the camera position and orientation, $\boldsymbol{r}(t)$ is the parameterized trajectory followed by the robot end-effector, and the integral is taken over the duration of a given trajectory. With this formulation, the problem of optimal camera placement reduces to finding $\boldsymbol{q}_{c}^{*}$ such that

$$
\psi\left(\boldsymbol{q}_{c}^{*}\right)=\max _{\boldsymbol{q}_{c} \in \mathcal{C}_{c}} \int w\left(\boldsymbol{r}, \boldsymbol{q}_{c}\right) d t .
$$

When other viewpoint constraints are taken into account, such as those given in [16], $\mathcal{C}_{c}$ would be restricted to the set of valid camera configurations.

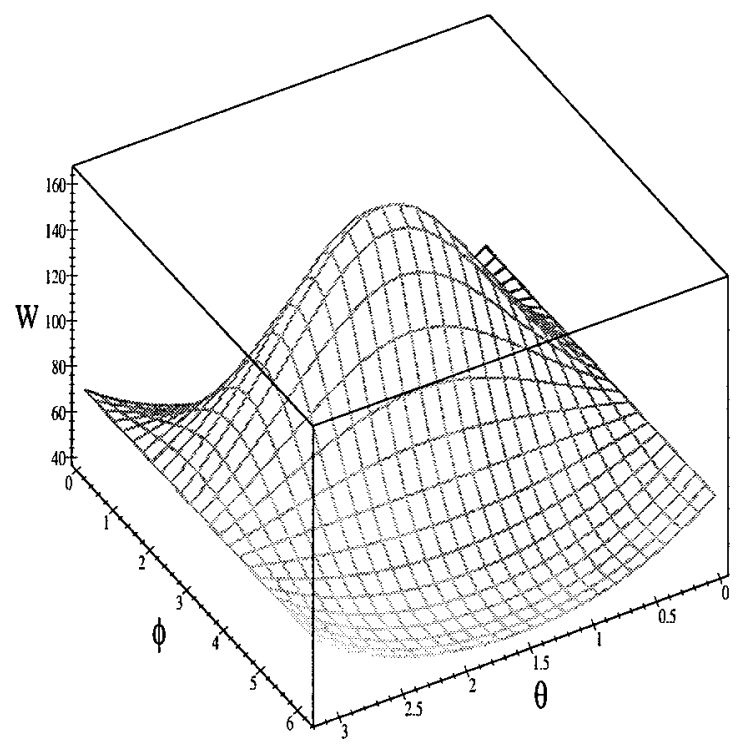

(a)

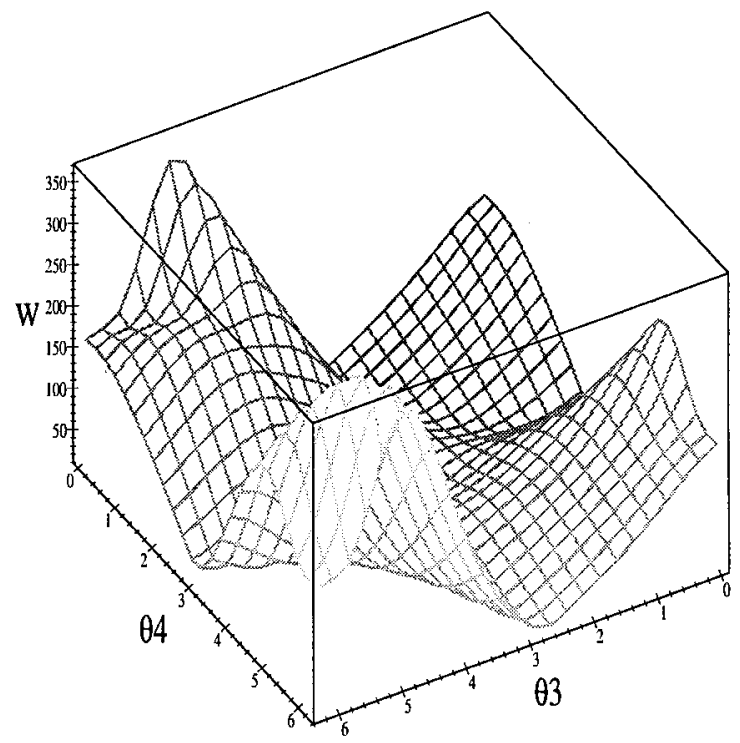

(b)

Fig. 7. Variation of the composite perceptibility/manipulability measure, $w=w_{v} \cdot w_{r}$, with changes in (a) camera configuration and (b) robot configuration.

Consider the problem of optimal camera placement for the case of the four-link planar robot setup in Fig. 4. Consider two different trajectories of the robot, obtained by varying $\theta_{4}$ as a function of the parameter $\tau$, as follows: a) $\theta_{4}=\sin (\pi \tau / 2)+\cos (\pi \tau / 2)$, and b) $\theta_{4}=\tan (\pi \tau / 3)$, while keeping the other joints fixed. Fig. 8 shows the plot of these two trajectories and the variation of the performance measure $\psi$ (obtained by integrating $w$ over the entire trajectory) as a variation of the camera parameter $\phi$. The integration was carried out numerically using the XMAPLE software. The optimal positions of the camera correspond to the maximas in the plotted curves and are shown in Fig. 8.

\section{B. Active Camera Trajectory Planning}

In this section, we consider the case when the camera is actively moved during the motion of the robot. Camera trajectory planning has many applications, particularly for repetitive operation of a robot 


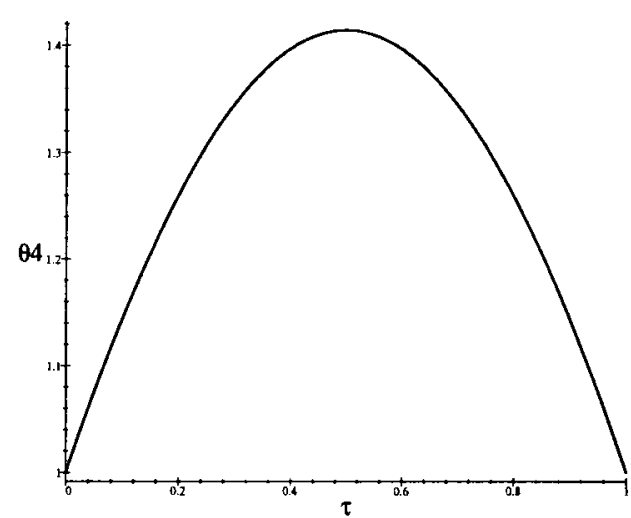

robot trajectory

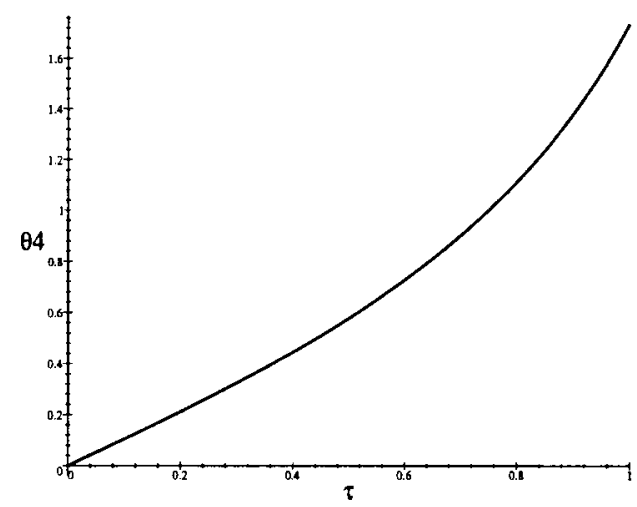

robot trajectory

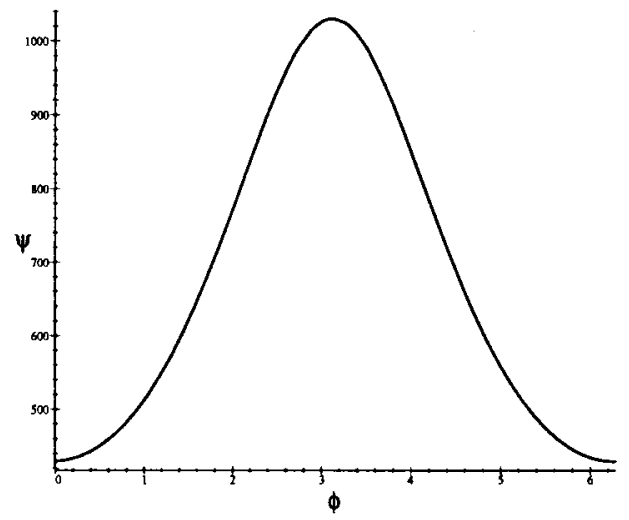

variation of cost $\left(\phi_{\text {optimal }}=3.35 \mathrm{rad}\right)$

(a)

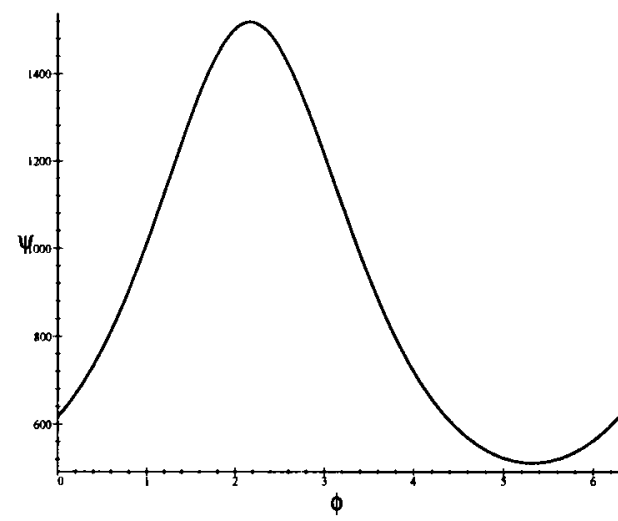

variation of cost $\left(\phi_{\text {optimal }}=2.23 \mathrm{rad}\right)$

(b)

Fig. 8. Finding the optimal camera position for given trajectories of the four-link planar robot.

in a very cluttered workspace, for example, in welding or painting in a car's interior. The implementation could involve two cooperating robot systems, with one "eye-in-hand" robot monitoring the operation of another robot performing a manipulation task. This is an example of active vision, which holds great promise in improving visual servoing as well other vision-based operation [12].

To optimize the camera trajectory we must find a camera trajectory, $\boldsymbol{q}_{c}(t)$, such that

$$
\psi\left(\boldsymbol{q}_{c}^{*}(\cdot)\right)=\max _{\boldsymbol{q}_{c}(\cdot)} \int w\left(\boldsymbol{r}, \boldsymbol{q}_{c}\right) d t .
$$

To ensure a physically realizable camera trajectory, kinematic constraints might have to be considered as well. In general, this class of problems can only be solved by constraining the class of trajectories that are permitted for the active camera.

For the following computed examples, to simplify the optimization procedure, we use a greedy algorithm that determines the locally best move of the camera at each time interval. In particular, if the robot and camera are in configurations $\boldsymbol{q}$ and $\boldsymbol{q}_{c}$, respectively, we select $\delta \boldsymbol{q}_{c}^{*}$ such that

$$
w\left(\boldsymbol{q}, \boldsymbol{q}_{c}+\delta \boldsymbol{q}_{c}^{*}\right)=\max _{\delta \boldsymbol{q}_{c}} w\left(\boldsymbol{q}, \boldsymbol{q}_{c}+\delta \boldsymbol{q}_{c}\right) .
$$

At each stage, the set of possible choices for $\delta \boldsymbol{q}_{c}$ is restricted to reflect the set of reachable positions when kinematic constraints are incorporated in the camera motion.
Consider the hand/eye setup of Fig. 4, where the camera is allowed to move only along a circle, defined by fixing $\theta$ and varying $\phi$. Fig. 9 shows the result of the active camera planning for two different robot trajectories with the restriction that at each step (unit of time) the movement of the camera is bounded $(0.05$ radians). The starting position of the camera in both the cases is $\phi=\pi / 4$. The two robot trajectories are plotted parametrically and the resulting active camera trajectories are shown that optimize the composite perceptibility/manipulability measure under the given motion constraints (Fig. 9).

\section{Simultaneous Camera/Robot Trajectory Planning}

In this section we consider the simultaneous optimization of the robot and camera trajectories with respect to the composite perceptibility/manipulability measure. To simplify the optimization procedure, we use a greedy algorithm that determines the locally best move of both the robot and the camera at each time interval. In particular, if the robot and camera are in configurations $q$ and $q_{c}$, respectively, we select $\delta \boldsymbol{q}_{c}^{*}$ and $\delta \boldsymbol{q}^{*}$ such that

$$
w\left(\boldsymbol{q}+\delta \boldsymbol{q}^{*}, \boldsymbol{q}_{c}+\delta \boldsymbol{q}_{c}^{*}\right)=\max _{\delta \boldsymbol{q}, \delta \boldsymbol{q}_{c}} w\left(\boldsymbol{q}+\delta \boldsymbol{q}, \boldsymbol{q}_{c}+\delta \boldsymbol{q}_{c}\right) .
$$

Kinematic and dynamic constraints are incorporated into the set of possible choices for $\delta \boldsymbol{q}$ and $\delta \boldsymbol{q}_{c}$ at each stage. A weighting factor could be used to determine the relative importance of optimizing $w$ 


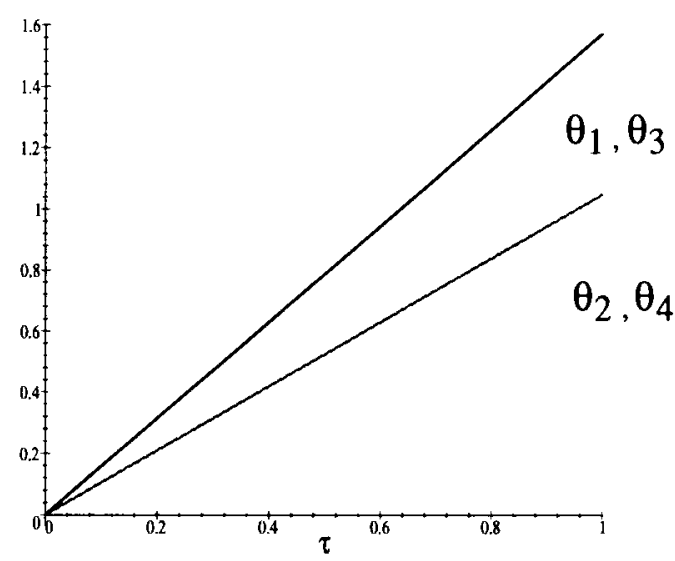

given robot trajectory

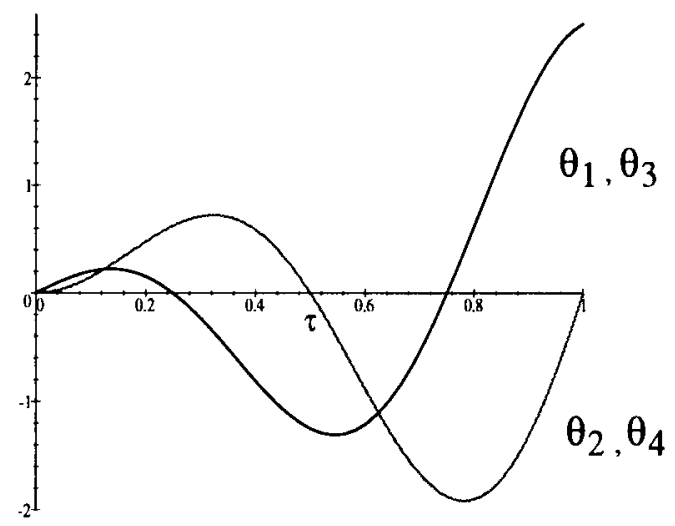

given robot trajectory

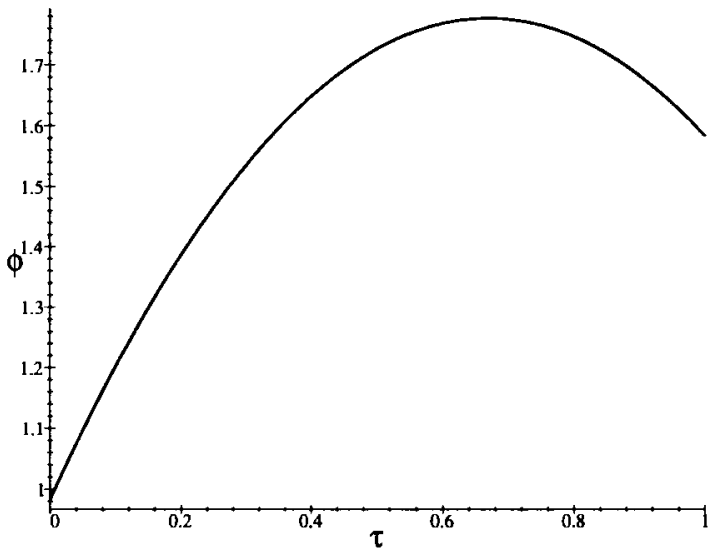

resulting active camera trajectory

(a)

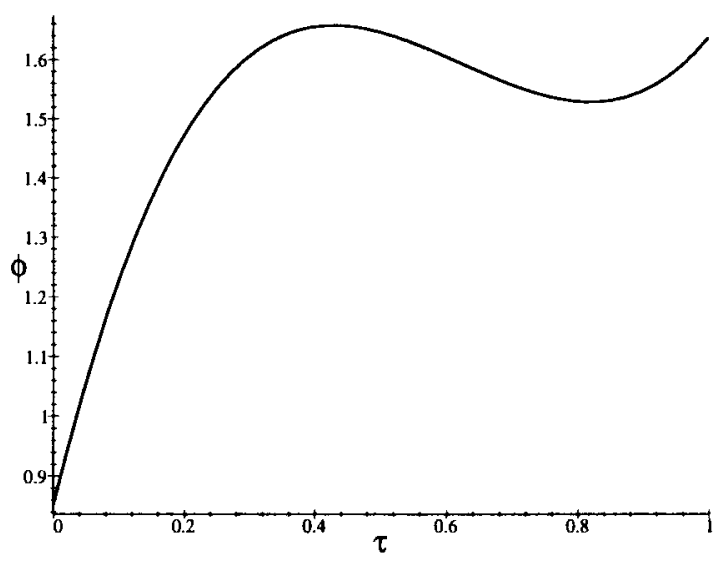

resulting active camera trajectory

(b)

Fig. 9. The result of active camera trajectory planning for optimizing the composite perceptibility/manipulability under given constraints, for two different trajectories of the four-link planar robot.

versus the cost of achieving the desired joint positions in terms of time or the total joint motion. The issue is basically efficiency in the robot trajectory planning versus the ease of achieving the visual servo control.

We consider the four-link planar robot. We will consider the case when either the camera $\phi$ and robot joint $\theta_{3}$ is used for improving the value of $w$. Because of the redundant degrees of freedom, the three joints $\theta_{1}, \theta_{2}, \theta_{4}$ will be used to both "compensate" for the joint $\theta_{3}$ while accomplishing the desired end-effector trajectory. In the two examples depicted in Fig. 10, the joint $\theta_{2}$ is given a prescribed trajectory, while motion of the joints $\theta_{1}, \theta_{4}$ is used to attain a desired end-effector trajectory. For brevity, the trajectories of $\theta_{1}$ and $\theta_{4}$ are not shown. For a simple linear trajectory of $\theta_{2}$, Fig. 10(a) shows the trajectories for $\theta_{3}$ and the camera parameter $\phi$ that optimize $w$ as discussed above. The maximum rate of change for both $\theta_{3}$ and $\phi$ was set to be 0.05 radians per unit step. Fig. 10(b) shows the analogous results for a more complicated trajectory of $\theta_{2}$.

\section{Critical Directions for Hand/Eye Motion}

The motion perceptibility measure, $w_{v}$, is a single scalar quantity that is designed to give a quantitative assessment of the overall perceptibility of robot motions. If motion in any direction is not observable, then $w_{v}=0$ (in this case $\boldsymbol{J}_{v}$ is singular). In many tasks, it is not important that the vision system be able to observe every direction of motion with high precision. Consider, for example, the peg-in-hole task illustrated in Fig. 11(a). A simple strategy is to align the peg with the hole, push the peg into the hole. To execute this strategy, it is important that the vision system be able to observe motion in directions parallel to the surface of the block, but it is much less critical that the vision system be able to precisely observe motions in the direction perpendicular to this surface. The motion perceptibility, $w_{v}$, is not sufficient to capture such distinctions.

We can use the singular value decomposition of $\boldsymbol{J}_{v}$ to assess the perceptibility of motions in particular directions. Using (2) and (9), we can determine the magnitude of the visual feature velocity for a particular motion $\dot{\boldsymbol{r}}$ as follows:

$$
\begin{aligned}
\boldsymbol{v} & =\boldsymbol{J}_{v} \dot{\boldsymbol{r}}=\boldsymbol{U} \Sigma\left[\begin{array}{llll}
\boldsymbol{v}_{1} & \boldsymbol{v}_{2} & \cdots & \boldsymbol{v}_{m}
\end{array}\right]^{T} \dot{\boldsymbol{r}} \\
& =\boldsymbol{U}\left[\begin{array}{llll}
\sigma_{1} \boldsymbol{v}_{1} & \sigma_{2} \boldsymbol{v}_{2} & \cdots & \sigma_{m} \boldsymbol{v}_{m}
\end{array}\right]^{T} \dot{\boldsymbol{r}} \\
& =\boldsymbol{U}\left[\begin{array}{c}
\sigma_{1} \boldsymbol{v}_{1} \cdot \dot{\boldsymbol{r}} \\
\sigma_{2} \boldsymbol{v}_{2} \cdot \dot{\boldsymbol{r}} \\
\vdots \\
\sigma_{m} \boldsymbol{v}_{m} \cdot \dot{\boldsymbol{r}}
\end{array}\right]
\end{aligned}
$$

Since $U$ is an orthogonal matrix,

$$
\begin{aligned}
& \|\dot{\boldsymbol{v}}\|=\left\|\left[\begin{array}{llll}
\sigma_{1} \boldsymbol{v}_{1} \cdot \dot{\boldsymbol{r}} & \sigma_{2} \boldsymbol{v}_{2} \cdot \dot{\boldsymbol{r}} & \cdots & \sigma_{m} \boldsymbol{v}_{m} \cdot \boldsymbol{r}
\end{array}\right]^{T}\right\| \\
& =\left(\left(\sigma_{1} \boldsymbol{v}_{1} \cdot \dot{\boldsymbol{r}}\right)^{2}+\left(\sigma_{2} \boldsymbol{v}_{2} \cdot \dot{\boldsymbol{r}}\right)^{2}+\cdots\left(\sigma_{m} \boldsymbol{v}_{m} \cdot \dot{\boldsymbol{r}}\right)^{2}\right)^{1 / 2} .
\end{aligned}
$$




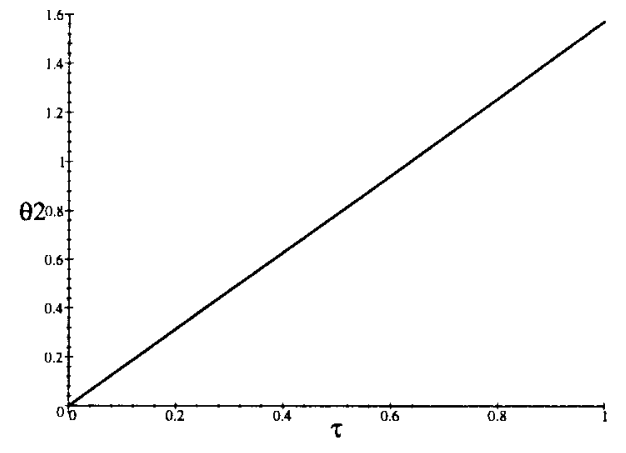

$$
\text { given robot motion, } \theta_{2}(\tau)
$$

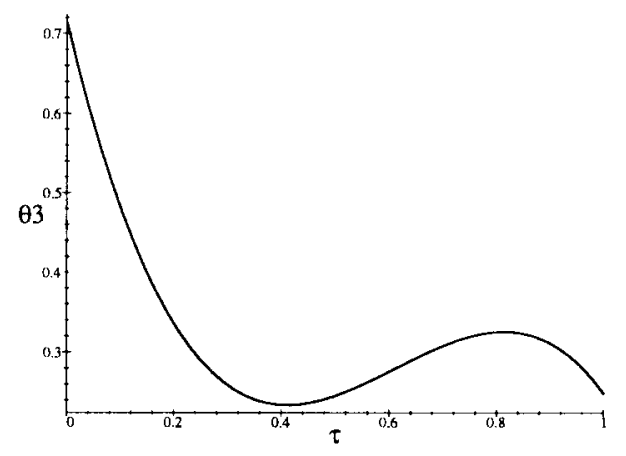

optimizing robot motion, $\theta_{3}(\tau)$

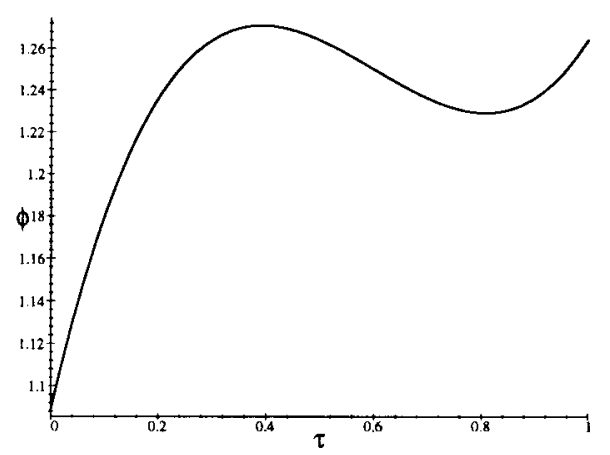

active camera motion, $\phi(\tau)$

(a)

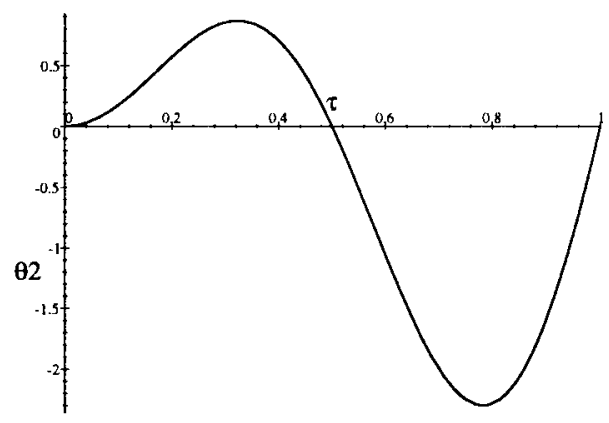

given robot motion, $\theta_{2}(\tau)$

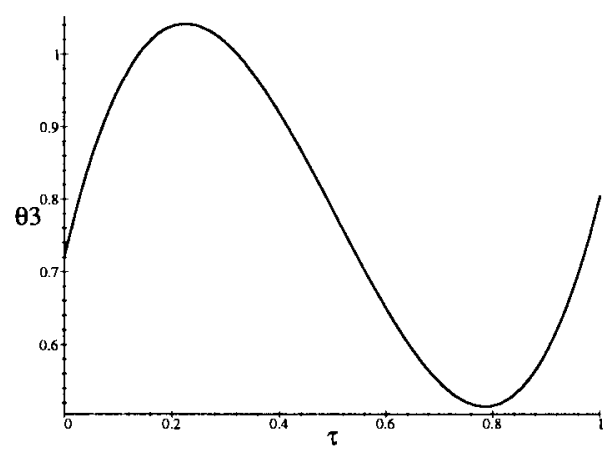

optimizing robot motion, $\theta_{3}(\tau)$

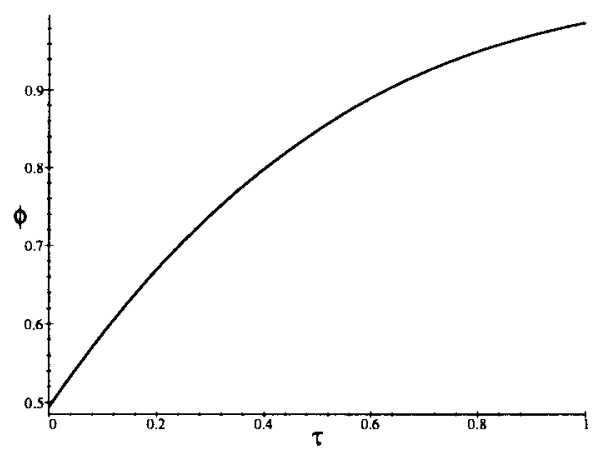

active camera motion, $\phi(\tau)$

(b)

Fig. 10. Results of simultaneous joint $\left(\theta_{3}\right)$ and camera $(\phi)$ trajectory for optimizing the composite perceptibility/manipulability measure for the given robot trajectory $\left(\theta_{2}\right)$.

By choosing $\dot{r}$ as a weighting vector that expresses the relative importance of the perceptibility of the different directions of robot motion, we can use (38) to evaluate the camera configuration. For the example peg-in-hole task, assuming that the hole is a perfect circle, we would let $\boldsymbol{r}=\left[\begin{array}{lll}0.5 & 0.5 & 0\end{array}\right]^{T}$, specified in the local frame of the hole; this implies that the motion along $x$ and $y$ directions are equally important while motion along the $z$ direction is not important for the task. The maximum for (38) will be achieved when $\boldsymbol{v}_{1}$ and $\boldsymbol{v}_{2}$ form a basis for the local $x-y$ plane for the hole, and when $\sigma_{1}$ and $\sigma_{2}$ are maximized. The value for $\sigma_{3}$ is irrelevant, since, as described above, for this task it is not important to observe motion perpendicular to the plane containing the hole. Fig. 11(b) shows the value of modified perceptibility measure, $v(38)$ as a function of $\theta$, the orientation of the block (about it's own $y$-axis). As can be seen in the figure, maxima is achieved when the face of the block is parallel to the camera image plane, which matches our intuition.

\section{E. Other Applications}

Besides those applications discussed above, there are a number of additional applications that are not restricted to visually controlled manipulation tasks. In this section, we briefly discuss a few of these.

One means of improving visual servoing is to select the right set of features from the many possible image features. In [5] for 


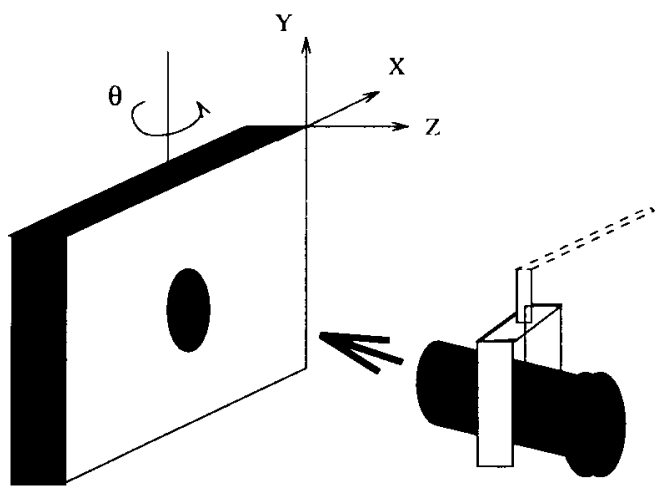

(a)

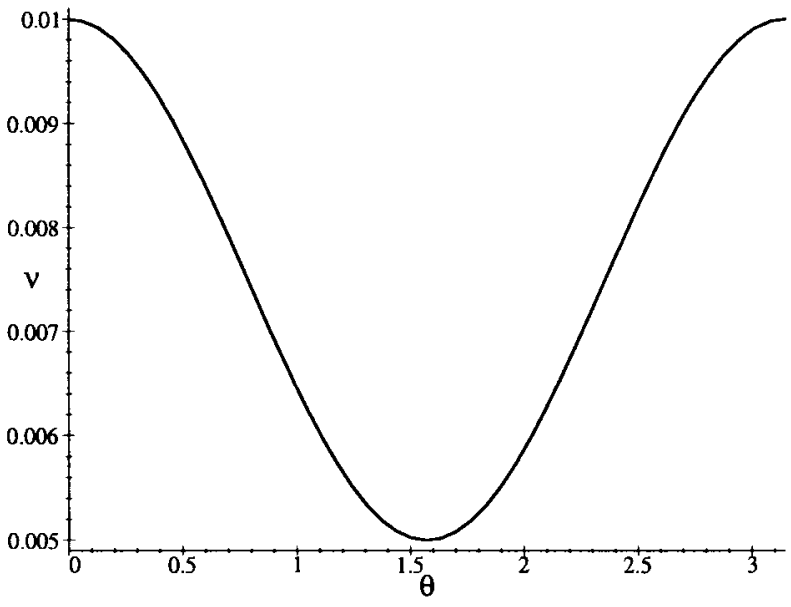

(b)

Fig. 11. (a) Example peg-in-hole task. (b) A plot of the modified motion perceptibility measure versus viewing angle $(\theta)$.

example, a method of feature selection was proposed that weighs several different criteria including photometric ones. The motion perceptibility measure can be factored into the feature selection process. The goal would be to select those features that will not get into "singular" configurations, where the change in the features becomes small or even zero with robot motion. One can formulate the corresponding optimization criteria in terms of the value of motion perceptibility.

Automated inspection tasks in manufacturing might involve highprecision measurement of part dimensions. For such tasks, in order to maximize the accuracy of a set of critical measurements made by the inspection system, it is beneficial to position the camera so that the image deviations in critical dimensions of the manufactured part are easily observed. The methods proposed in Section D are particularly well suited for evaluating camera positions under these circumstances.

In remote teleoperation applications, a user controls the robot's motions using some interface device (e.g., a track ball, joy stick, or power glove), while watching a robot on a video display that is provided by a remote supervisory camera in the robot's environment. In this case, the motion perceptibility measure can be used to determine the optimal location for the supervisory the camera, so that the human operator can best observe the relevant degrees of freedom of robot motion.

\section{Discussion AND CONCLUSIONS}

In our discussion of motion perceptibility, we have restricted our attention to geometric considerations. Non-geometric criteria (e.g., feature detectability) may be combined with motion perceptibility, for example by taking a weighted sum of normalized costs [5]. A survey of other criteria can be found in [16], which deals with automatically selecting different camera parameters for a given task.

Motion perceptibility represents a single scalar measure, corresponding to the volume of the motion perceptibility ellipsoid at a particular configuration. Other scalar measures could have been used as well, for example, the ratio of the minimum diameter to the maximum diameter of the motion perceptibility ellipsoid. In the related context of manipulation, [6] gives a very good discussion for the physical meaning and shortcomings of the various dexterity measures.

Motion perceptibility is a local property of the relative hand/eye configurations. If a single camera is to be used for multiple tasks, for example, exploring [16] and task monitoring [8], [20], then global optimization problems analogous to the ones posed in Section VI would need to be solved. Such optimizations may be quite difficult to perform. Thus, one avenue for future research is to derive approximate techniques that give the most importance to the critical parameters in determining the camera position at a given time.

Despite these limitations, the measure of motion perceptibility that we introduce captures a very basic property of relative camera position in a hand/eye setup. It has an intuitive meaning and provides a formal basis for positioning a camera relative to a robot for controlling an active camera, visual servo control, or trajectory planning. The composition of the motion perceptibility with the classical manipulability measure further helps in dealing with various hand/eye optimization problems. We used both a redundant and a nonredundant robot to illustrate these potential applications of the motion perceptibility measure.

\section{ACKNOWLEDGMENT}

The authors would like to thank the anonymous reviewers whose insightful comments were a great help in improving the presentation of the paper.

\section{REFERENCES}

[1] P. K. Allen, A. Timcenko, B. Yoshimi, and P. Michelman, "Automated tracking and grasping of a moving object with a robotic hand-eye system," IEEE Trans. Robot. Automat., vol. 9, pp. 152-165, 1993.

[2] A. Castano and S. Hutchinson, "Visual compliance task-directed visual servo control," IEEE Trans. Robot. Automat., vol. 10, pp. 334-342, 1994.

[3] P. I. Corke, "Visual control of robot manipulators - A review," in Visual Servoing, K. Hashimoto, Ed. Singapore: World Scientific, 1993, pp. 1-32.

[4] B. Espiau, F. Chaumette, and P. Rives, "A new approach to visual servoing in robotics," IEEE Trans. Robot. Automat., vol. 8, pp. 313-326, 1992.

[5] J. T. Feddema, C. S. G. Lee, and O. R. Mitchell, "Weighted selection of image features for resolved rate visual feedback control," IEEE Trans. Robot. Automat., vol. 7, pp. 31-47, 1991.

[6] J.-O. Kim and P. K. Khosla, "Dexterity measures for design and control of manipulators," in Proc. IEEE/RSJ Int. Workshop on Intell. Robot. Syst., 1991, pp. 758-763.

[7] A. J. Koivo and N. Houshangi, "Real-time vision feedback for servoing robotic manipulator with self-tuning controller," IEEE Trans. Syst., Man, Cybern., vol. 21, pp. 134-142, 1991.

[8] C. Laugier, A. Ijel, and J. Troccaz, "Combining vision-based information and partial geometric models in automatic grasping," in Proc. IEEE Int. Conf. Robot. Automat., 1990, pp. 676-682.

[9] Y. Nakamura, Advanced Robotics: Redundancy and Optimization. Reading, MA: Addison-Wesley, 1991.

[10] B. J. Nelson and P. K. Khosla, "The resolvability ellipsoid for visual servoing," in Proc. IEEE Conf. Comput. Vision and Pattern Recog., 1994, pp. 829-832.

[11] N. P. Papanikolopoulos, P. K. Khosla, and T. Kanade, "Visual tracking of a moving target by a camera mounted on a robot: A combination 
of vision and control," IEEE Trans. Robot. Automat., vol. 9, pp. 14-35, 1993.

[12] R. Sharma, "Active vision for visual servoing: A review," in IEEE Workshop on Visual Servoing: Achievements, Applications and Open Problems, May 1994.

[13] R. Sharma and H. Sutanto. "A framework for robot motion planning with sensor constraints," IEEE Trans. Robot. Automat., vol. 13, pp. 61-73, Feb. 1997.

[14] S. B. Skaar, W. H. Brockman, and R. Hanson. "Camera-space manipulation," Int. J. Robot. Res., vol. 6, no. 4, pp. 20-32, 1987.

[15] H. Sutanto and R. Sharma. "Global performance evaluation of image features for visual servo control," J. Robot. Syst., vol. 13, no 4, pp. 243-258, Apr. 1996.

[16] K. Tarabanis and R. Y. Tsai, "Sensor planning for robotic vision: A review," in Robotics Review 2, O. Khatib, J. J. Craig, and T. LozanoPérez, eds. Cambridge, MA: MIT Press, 1992.

[17] L. E. Weiss, A. C. Sanderson, and C. P. Neuman, "Dynamic sensorbased control of robots with visual feedback," IEEE J. Robot. Automat., vol. 3, pp. 404-417, 1987.

[18] T. Yoshikawa, "Analysis and control of robot manipulators with redundancy," in Robotics Research: The First Int. Symp. Cambridge, MA MIT Press, 1983, pp. 735-747.

[19] B. Yoshimi and P. K. Allen, "Active, uncalibrated visual servoing," in Proc. IEEE Int. Conf. Robot. Automat., San Diego, CA, May 1994, pp. $156-161$.

[20] J. Y. Zheng, Q. Chen, and A. Tsuji, "Active camera guided manipulation," in Proc. IEEE Int. Conf. Robot. Automat,, 1991, pp. 632-638.

\section{Impedance Restrictions on Independent Finger Grippers}

\author{
M. E. Brokowski and Michael Peshkin
}

\begin{abstract}
The impedance matrices of independent point fingers of a multifingered gripper map to the impedance matrix of a grasped workpart. We find that in a planar geometry, three fingers are enough to allow an unrestricted range of workpart impedances, if finger impedances are selectable. In a spatial geometry however, five fingers are necessary for the broadest range of workpart impedances, and even so there is one impedance matrix that a workpart cannot attain regardless of the number of fingers that grasp it. We find this "unattainable" impedance matrix. We also characterize the impedance restrictions on workparts grasped with fewer than five spatial or three planar fingers.
\end{abstract}

Index Terms - Compliance, grippers, impedance, multifingered, restrictions, robot.

\section{INTRODUCTION}

Force-guided assembly allows a robot to use the forces generated during an assembly operation to guide the operations successful completion. One implementation of force-guided assembly utilizes impedance control, wherein a workpart's impedance is specified such that forces resulting from errors in positioning are mapped to motions that reduce the errors [14].

Consider a multifingered gripper where each finger can have a specifyable impedance characteristic. A grasped workpart will have an effective impedance characteristic that is a function of the

Manuscript received May 3, 1995; revised August 1, 1996. This paper was recommended for publication by Associate Editor J. C. Trinkle and Editor A. Goldenberg upon evaluation of the reviewers' comments.

The authors are with the Department of Mechanical Engineering, Northwestern University, Evanston, IL 60208 USA.

Publisher Item Identifier S 1042-296X(97)05904-1. impedances of the fingers and of the grasp geometry (i.e. the points where the fingers contact the workpart). Often, we wish to confer a particular impedance characteristic on a workpart by specifying particular impedance characteristics for the fingers that grasp it. For linear admittance/impedance*, this means that the impedance matrices of the fingers map to an effective impedance matrix of the grasped workpart.

The goal of this paper is to explore what sorts of impedance properties a grasped workpart can have when gripped with a given number of fingers whose impedance we can control.

\section{A. Language}

Suppose that we can choose each element of each fingers damping matrix to be whatever we want. If we can confer a particular damping matrix on the workpart by choosing an appropriate set of these finger damping matrices, then we say that the workpart damping matrix is attainable.

More formally, let $\left\{\boldsymbol{D}_{i}\right\}, i=1 \cdots n$, be the set of $n$ damping matrices corresponding to $n$ fingers grasping some workpart and let the damping matrix of the grasped workpart as a function of that set be $\boldsymbol{D}_{\text {workpart }}=f\left(\left\{\boldsymbol{D}_{i}\right\}\right)$. Then $\boldsymbol{D}_{\text {workpart }}$ is attainable if and only if

$$
\exists\left\{\boldsymbol{D}_{i}\right\} \ni \boldsymbol{D}_{\text {workpart }}=f\left(\left\{\boldsymbol{D}_{i}\right\}\right) .
$$

Further, if all workpart damping matrices are attainable, each attained by choosing an appropriate $\left\{\boldsymbol{D}_{i}\right\}$, we say that $f$ is a full rank mapping.

\section{B. Scope of This Paper}

Using this language, we will determine two things. First, we will determine the number of fingers that must grasp an workpart in order to achieve a full rank mapping from the finger damping matrices to the damping matrix of the workpart.

We will also determine the limitations on the attainable damping/accommodation matrices of the grasped workpart when it is grasped by fewer than the number of fingers needed for a full rank mapping. That is, when we have too few fingers to get any workpart damping/accommodation matrix that we want, which ones can we still get?

We do not address the inverse problem of designing finger accommodations for a particular desired workpart accommodation. In this paper, the damping matrices of the fingers are assumed known, it is the damping characteristics of the grasped workpart that we wish to determine.

We also do not determine optimal grasp geometry. The positions of the fingers on the workpart are unrestricted, but they are assumed to be known.

\section{Assumptions}

- The robot is rigid outside of its fingers. That is, we are not treating any damping of the robot which cannot be accounted for in the fingers. In addition, the workpart is a rigid, massless body, a reasonable approximation for workparts which are relatively stiff and light compared to the fingers which hold them.

- We speak of damping/accommodation properties, though generalization to impedances, including stiffness and mass, is totally analogous for our rigid massless workparts. 